\title{
Counteracting the Effects of GNSS Jamming in a Maritime Multi-Target Scenario by Fusing AIS with Radar Data
}

\author{
Gregor Siegert, Paweł Banyś, Julian Hoth and Frank Heymann \\ Department of Nautical Systems, German Aerospace Center (DLR)
}

\section{BIOGRAPHIES}

Gregor Siegert is a research fellow at the German Aerospace Center (DLR), in the Department of Nautical Systems of the Institute of Communications and Navigation. In 2010 he received his Diploma degree in electrical engineering from the University of Technology Ilmenau in Germany. Before joining DLR in 2015, he worked in the field of SatCom-On-The-Move and OverThe-Air RF testing at the Fraunhofer Institute for Integrated Circuits. Currently, his research is focused on cooperative maritime traffic situation assessment being particularly interested in distributed sensor fusion and target tracking.

Paweł Banyśs holds a master's degree in finance and banking and an engineer's degree in geodesy and cartography. Between 2001 and 2010 he was employed at different IT companies as network and Linux administrator. He also cooperated with the Maritime University of Szczecin on a vessel traffic safety project. Since 2010 he has been working at the DLR Department of Nautical Systems in the field of AIS and maritime traffic systems.

Julian Hoth is a research associate at the German Aerospace Center (DLR) in the Department of Nautical Systems. He received a master's degree in mechanical engineering and a doctoral degree from the University of Duisburg-Essen, Germany. Before joining DLR in 2016, Julian worked in the area of underwater navigation and underwater imagery at the Chair of Mechanics and Robotics of the University of Duisburg-Essen. His current research is focused on radar target detection and tracking.

Frank Heymann received a $\mathrm{PhD}$ in physics from the University of Bochum in collaboration with the European Southern Observatories (ESO). From 2010 until 2012 he has worked in the field of active galactic nuclei in astrophysics as a postdoctoral researcher at the University of Kentucky. In 2012 he joined the DLR Institute of Communications and Navigation as a research associate in the field of maritime navigation. Since 2014 he is the group leader of the group Traffic Systems in the Department of Nautical Systems.

\begin{abstract}
With the introduction of the Automatic Identification System (AIS) in 2004, GNSS became a cornerstone for traffic situation assessment in the maritime domain. In this paper we study the impact of GNSS jamming on AIS and explore means of compensating potential AIS outages by fusing AIS with a GPS independent sensor such as radar. An IMM-JPDA filter was designed for multi-target tracking in presence of radar measurements originating from clutter. To demonstrate the working principle of the proposed scheme a dedicated measurement campaign was conducted in the Baltic Sea. In total three vessels were involved, two of them performing highly dynamic maneuvers at sea with the third vessel being anchored in the center of these maneuvers. This static vessel did not only monitor the scene by her radar, but was also equipped with a commercial high power GPS jammer.
\end{abstract}

\section{INTRODUCTION}

Over the past decades, the need for an accurate and resilient maritime traffic situation assessment (MTSA) has been increasingly growing due to a variety of reasons: the ever increasing global trade constantly calls for ships greater in size and numbers, considering that about $80 \%$ of the traded goods are traversed via sea [1]. Simultaneously, potential threats to the security at sea, such as piracy or terrorism have become an undoubted risk to secure and efficient shipping along the international waterways and harbors. In addition, it is a stringent necessity to detect abnormal vessel behavior, to prevent harm to humans and nature. Migrant vessels or illegal fishing activities are merely two prominent examples. The dominating source for situation assessment 
in the maritime domain has been and will be the marine radar, which is still the primary sensor for collision avoidance. However, with the introduction of the Automatic Identification System (AIS) in 2004 by the International Maritime Organization (IMO) a giant leap was taken towards an improved TSA. The AIS can be understood as supplemental service allowing vessels to exchange dynamic, static and voyage related data among each other via VHF broadcasting channels. The dynamic AIS message essentially contains the vessel's position, course over ground (COG) and speed over ground (SOG), all obtained from an internal or attached GPS receiver. Besides the intentional abuse of its cooperative nature, being analyzed in [2], the dependency on an external system like GPS is also a potential threat to the functioning of AIS. In fact, the phenomenon of GNSS jamming would heavily affect the system's reliability. In contrast to the common approach of counteracting GNSS jamming at the receiver side, we propose in this work to mitigate the effects by fusing the AIS data with a sensor of complementary nature, the radar. This approach exploits the typical behavior of the AIS to switch to default values in case the positions cannot be obtained from GPS. Additionally, a more robust traffic situation picture can be realized without the requirement for any additional on-board equipment. In the literature various approaches have been published to augment maritime surveillance or collision avoidance systems, mostly based on fusing radar with additional sensors like laser in [3] or with multiple radars for exploiting aspect angle diversity as in [4]. The matter of AIS and radar fusion was mainly addressed for anomaly detection, e.g., based on multi hypothesis tests in [5] or by exploiting historical traffic route knowledge for Synthetic Aperture Radar (SAR)/AIS fusion in [6]. Within these approaches either the associations between AIS and radar targets are assumed to be known or non-real time SAR imagery is incorporated for large scale maritime surveillance. In contrast to that, we propose to treat each AIS message and radar image frame as individual measurement input to a multi-sensor, multi-target tracking framework yielding target state estimates for the current time. Consequently, individual sensor outages, e.g., from AIS due to GNSS jamming, can be compensated much better in case the other sensor is still capturing the target motion in its measurement domain. The challenge of multitarget tracking (MTT) in the presence of clutter measurements has been addressed in numerous frameworks, roughly divided in families of enumerative and non-enumerative algorithms. The former explicitly evaluates data associations ranging from rather simple Nearest Neighbor (NN) association rules to more complex schemes such as Joint Probabilistic Data Association (JPDA) or Multi Hypothesis Tracking (MHT), with both being well-described in [7] and [8], respectively. The research on non-enumerative algorithms also led to the introduction of Random Finite Set (RFS) theory to MTT, such as Probability Hypothesis Density (PHD) or Cardinalized PHD (CPHD) filtering as described in [9] and [10]. Considering the given challenge of on-board fusion of two sensors, i.e., radar and AIS, with multiple but a limited number of targets to be tracked, the JPDA framework was chosen as it promises to provide good performance at fair complexity in comparison to NN and MHT algorithms [11]. However, an additional challenge to the tracking task was identified in the wide range of target dynamics to be expected in the maritime world. In principle, vessels are either undergoing straight path or turn-maneuver motion, whereas the maneuverability itself highly depends on the type of vessel. In fact, a single motion model with no additional control input will hardly be able to follow a target with rapidly changing dynamics. A common way to deal with these challenges is to deploy an Interacting Multiple Model (IMM) filter, first described in [12], which is tracking the state of a single target by running a bank of Kalman Filters in parallel, each being conditioned on a different motion model. The IMM filter provides a scheme to combine the outputs of the individual trackers in a probabilistic sense. The coupling of both algorithms, that is the IMM with the JPDA, was first presented in [13], whereas [14] also considered the use of multiple sensors in a combined framework.

The remaining document is structured as follows. At first, the methodology for multi-target tracking using multiple sensors in the presence of clutter measurements will be depicted in more detail. This is followed by a detailed discussion of the results obtained from applying the designed filter to real-world measurement data, which captured different challenges such as strong Global Navigation Satellite System (GNSS) interference and fast maneuvering targets. The paper is concluded including an outlook to future work in the last section.

\section{FUSING AIS WITH RADAR IN AN MTT FRAMEWORK}

The main objective in this work is to present a methodology yielding a robust and complete picture of the current traffic situation despite outages of individual sensors such as AIS. Plainly speaking, traffic situation assessment mainly deals with the challenge of detecting and tracking all objects in a certain area of interest, which is generally referred to as multi-target tracking. By incorporating data from multiple sensors with complementary characteristics the overall tracking is expected to be more robust against individual sensor failure. In this section the applied MTT framework, an IMM-JPDA filter, will be presented in more detail, also depicting the procedure for target candidate extraction from radar images.

\section{General dynamic and measurement model}

For tracking vessels of various types, we assume to propagate the state vector $\mathbf{x}_{k-1}^{t}$ of the $t^{t h}$ target to the next time frame $k$ through a non-linear motion model following the notation of

$$
\mathbf{x}_{k \mid k-1}^{t}=f^{i}\left(\mathbf{x}_{k-1}^{t}, \epsilon_{k}^{q}\right)
$$

where $\epsilon_{k}^{q} \sim \mathcal{N}\left(\mathbf{0}, \Sigma=\mathbf{Q}_{k}^{i}\right)$ and no further control input given. To distinguish between different dynamic models in the upcoming 
sections, we introduce the superscript $i$ to the non-linear function $f^{i}(\cdot)$. The predicted state estimate $\mathbf{x}_{k \mid k-1}^{t}$ will be corrected by evaluating the residual between the actual measurement $\mathbf{z}_{k}^{s}$ of sensor $s$ associated to the $t^{\text {th }}$ target and the predicted measurement for that sensor following the general formulation of

$$
\hat{\mathbf{z}}_{k \mid k-1}^{s}=h^{s}\left(\mathbf{x}_{k \mid k-1}^{t}, \boldsymbol{\epsilon}_{k}^{r}\right),
$$

with $\boldsymbol{\epsilon}_{k}^{r} \sim \mathcal{N}\left(\mathbf{0}, \Sigma=\mathbf{R}_{k}^{s}\right)$. In the remainder of this section, we will define the set of dynamic and measurement models that constitute the IMM-JPDA framework for multi-sensor, multi-target tracking.

\section{IMM-JPDA framework using UKF}

Considering the inherent trade-off between complexity and tracking performance the JPDA framework was chosen, being combined with an IMM filter to capture different target dynamics. Being first introduced in [15], the JPDA filter is subject to several assumptions. Most importantly for our application, the finite set of targets to be tracked is assumed to be known, i.e., neither track initialization nor track pruning is covered by the standard formulation of JPDA. These restrictions, however, are tolerable in our specific case of fusing radar target candidates with vessels visible in AIS. In fact, the number of targets to be tracked as well as their initial states are known from AIS within the considered measurement scenario. The key feature of the JPDA is the computation of conditional probabilities of joint association events

$$
A(k)=\bigcap_{j=1}^{M} A_{j t}(k)
$$

with respect to the current time $k$, in which $A_{j t}(k)$ represents the event of the $j^{\text {th }}$ measurement originating from target $t$, with $1 \leq j \leq M$ and $0 \leq t \leq N$. In this context, $M$ refers to the number of measurements at time $k, N$ to the number of known targets and $t_{j}$ is the target index the $j^{t h}$ measurement is associated to. With $t=0$ the specific case of a measurement originating from clutter is also being considered. This means, in contrast to a simple NN association rule, the JPDA also accounts for situations in which a single measurement can be assigned with a certain likelihood to multiple targets at the same time. In order to trace the different joint association events one usually constructs feasibility matrices $\mathbf{F}_{i}$; a set of binary matrices of dimensionality $M \times(N+1)$, with the first column index referring to the clutter origin. Such a matrix is subject to the following constraints:

1. A measurement can only originate from either clutter or a single target.

2. A target may be the origin of only one measurement at maximum, whereas the number of measurements originating from clutter is not limited to one.

These rules are typically expressed in the measurement and target association indicators $\tau_{j}(A(k))$ and $\delta_{t}(A(k)$, defined as

$$
\tau_{j}(A(k))=\left\{\begin{array}{ll}
1, & t_{j} \geq 1 \\
0, & t_{j}=0
\end{array}, \quad \delta_{t}(A(k))=\left\{\begin{array}{ll}
1, & t_{j}=t \text { for some } j \\
0, & t_{j} \neq t \text { for all } j
\end{array} .\right.\right.
$$

Using Bayes' rule, the final expression for the probability of a joint event $A$ given all measurements up to time $k$ is given as

$$
P\left\{A(k) \mid \mathcal{Z}^{k}\right\}=\frac{1}{c} \prod_{j}\left\{\lambda^{-1} f_{t_{j}}\left[\mathbf{z}_{j}(k)\right]\right\}^{\tau_{j}} \prod_{t}\left\{\left(P_{d}^{t}\right)^{\delta_{t}}\left(1-P_{d}^{t}\right)^{1-\delta_{t}}\right\} .
$$

Note, that the definition above is only valid for the parametric version of the JPDA in which the probability mass function (pmf) of the number of false (or clutter) measurements is modeled as Poisson distribution that depends on $\lambda$, i.e., the spatial density of false measurements. The probability of a target to be detected by a given sensor is expressed in $P_{d}^{t}$; we omit the superscript $t$ for the remainder of this work, assuming a constant target detection probability per sensor. Details can be found in [15] and [7]. In case the measurement $j$ is assigned to target $t$, the corresponding likelihood $f_{t_{j}}\left[\mathbf{z}_{j}(k)\right]$ in Eq. 4 is obtained from a normal distribution

$$
f_{t_{j}}\left[\mathbf{z}_{j}(k)\right]=\mathcal{N}\left[\mathbf{z}_{j}(k) ; \hat{\mathbf{z}}_{t_{j}}(k \mid k-1), \mathbf{S}_{t_{j}}(k)\right],
$$

that is set up around the predicted sensor measurement $\hat{\mathbf{z}}_{t_{j}}(k \mid k-1)$ and its covariance $\mathbf{S}_{t_{j}}(k)$. The factor $1 / c$ in Eq. 4 is a mere normalization constant.

It can easily be seen that the considerations of all joint events results in a combinatoric growth in complexity for more measurements and more targets. To narrow down the initial set of feasibility matrices a validation matrix $\mathbf{V}$ is usually computed first, which is also a binary matrix of dimensionality $M \times(N+1)$ representing all possible associations between measurements and targets. If the single matrix element $v_{m t}$ is either set to 0 or 1 depends on classical validation gating, i.e., the condition of

$$
\left[\mathbf{z}_{j}(k)-\hat{\mathbf{z}}_{t_{j}}(k \mid k-1)\right]^{T} \mathbf{S}_{t_{j}}^{-1}(k)\left[\mathbf{z}_{j}(k)-\hat{\mathbf{z}}_{t_{j}}(k \mid k-1)\right]<\gamma
$$




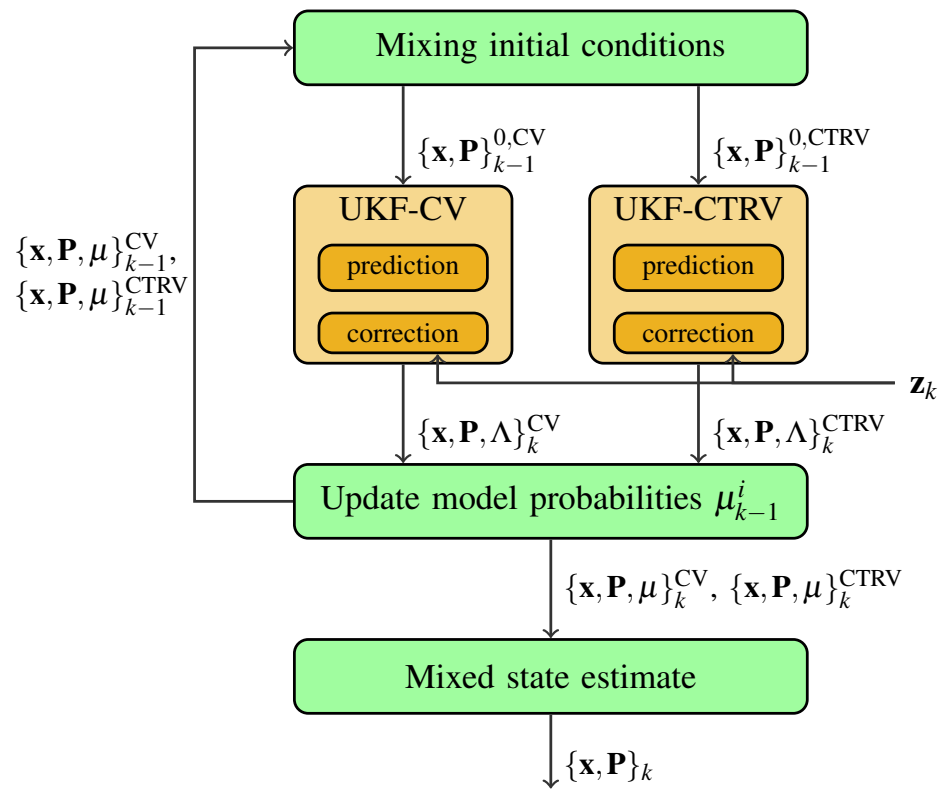

Figure 1: Simplified block diagram of the IMM filter running two UKFs conditioned on either a CV or CTRV dynamic model. Prior to each prediction-correction cycle the initial conditions of the individual UKFs are updated. The sensor measurement $\mathbf{z}_{k}$ is inferred to each of the filters to update the predicted target state. Based on the target state estimates (i.e., their mean $\mathbf{x}_{k}^{i}$ and covariance $\mathbf{P}_{k}^{i}$ ) and the corresponding likelihoods $\Lambda_{k}^{i}$, with $i \in\{\mathrm{CV}, \mathrm{CTRV}\}$, the model probabilities $\mu_{k}^{i}$ are updated. In the last stage, the combined target state estimate, i.e., its mean and covariance, is computed.

needs to be fulfilled for $v_{m t}$ to become 1 . Similar to Eq. 5 the volume $V$ of this gate depends on the covariance $\mathbf{S}_{t_{j}}^{-1}(k)$ of the predicted measurement. Despite this pre-selection of possible association events, various additional flavors of the JPDA filter were introduced aiming at the reduction of combinatoric complexity (see [11] for a comprehensive comparison).

In this work, we use an extension to classical JPDA filtering known as IMM-JPDA filter. The IMM was introduced by [12] to adopt to quickly changing target dynamics by considering a finite set of kinematic models that run in parallel. In contrast to hard switching schemes, the IMM weighs the different target state estimates based on the likelihood of each model to explain the current measurement data. The mode transition is thereby governed by an underlying Markov chain. The application of IMM filtering to maritime target tracking has already been shown to be beneficial in [16]. In our case, we consider a set of two dynamic models to capture either straight path or turning maneuver based motion. For the former a Constant Velocity (CV) model was designed, whereas the Constant Turn Rate Velocity (CTRV) model is supposed to fit best to the latter. The corresponding target state vectors are defined as

$$
\mathbf{x}_{k}^{\mathrm{CV}}=\left[p_{e, k}, p_{n, k}, \psi_{k}, v_{k}\right]^{T}, \quad \mathbf{x}_{k}^{\mathrm{CTRV}}=\left[p_{e, k}, p_{n, k}, \psi_{k}, v_{k}, \dot{\psi}_{k}\right]^{T},
$$

with $\left\{p_{e, k}, p_{n, k}\right\}$ being the $2 \mathrm{D}$ position coordinates in the local ENU frame, $\psi_{k}$ the course over ground, $v_{k}$ the speed over ground and $\dot{\psi}_{k}$ the turn rate at time $k$. The uncertainty within the models is expressed in

$$
\mathbf{Q}_{k}^{\mathrm{CV}}=\left[\begin{array}{cc}
\sigma_{\psi}^{2} & 0 \\
0 & \sigma_{v}^{2}
\end{array}\right], \quad \mathbf{Q}_{k}^{\mathrm{CTRV}}=\left[\begin{array}{cc}
\sigma_{v}^{2} & 0 \\
0 & \sigma_{\omega}^{2}
\end{array}\right] .
$$

The detailed definitions of the process models $f^{i}(\cdot)$ for CV and CTRV can be found in [17]. In many applications it can be beneficial to parameterize the different models in such a way that one acts more conservatively than the other. Applied to our case, the CV model is set to adopt to changes very slowly, i.e. the variances in $\mathbf{Q}_{k}^{C V}$ are rather small. Vice versa, more uncertainty is allowed to the CTRV yielding a model more flexible to rapid changes. Additionally, careful attention needs to be paid to the augmentation of state vectors of different dimensions. In this paper we follow a strategy described in [18] for unbiased mixing of different process models. In contrast to the common formulation of either IMM or JPDA, which both use Extended Kalman Filtering (EKF) to adopt to non-linearities in the dynamic models, we deploy the Unscented Kalman Filter (UKF) instead (see [19]). It turns out that due to the sigma point sampling approach the UKF is more robust against non-linearities induced by the radar measurement update equation, whereas the approximation to a first-order Taylor series expansion within the EKF was found to diminish its performance (see [4] for discussion). Figure 1 depicts the plain IMM filter for a single sensor measurement update and two UKFs running in parallel for tracking a single target. The combination of IMM and JPDA filtering schemes to a well-defined framework was initially proposed by [12] and extended to the multi-sensor case in [14]. In the end, a recursive 
step-by-step algorithm was derived fusing the asynchronous measurements from different sensors sequentially. The final state update equation for the $t^{\text {th }}$ target tracked in mode $i \in\{\mathrm{CV}, \mathrm{CTRV}\}$ becomes

$$
\mathbf{x}_{k}^{t, i}=\beta_{0 t}^{i} \mathbf{x}_{k \mid k-1}^{t, i}+\sum_{j=1}^{M_{t, k}} \mathbf{x}_{k \mid k}^{t, i}(j) \beta_{j t}^{i}
$$

with $M_{t, k}$ being the number of validated measurements for target $t$ and $\mathbf{x}_{k \mid k}^{t, i}(j)$ the UKF target estimate conditioned on the $j^{t h}$ measurement at time $k$. The weights $\beta_{j t}^{i}$ are interpreted as association probabilities following the convention in [4], with

$$
\begin{aligned}
& \beta_{0 t}^{i}=P\{\text { none of the measurements is originating from target } t\} \\
& \beta_{j t}^{i}=P\left\{\text { the } j^{\text {th }} \text { measurement origins from target } t\right\} .
\end{aligned}
$$

\section{Target candidate extraction from radar images}

In order to fuse radar with AIS position data, target candidates need to be detected and extracted from radar first to feed them to the filter as measurement updates. The utilized approach to extract radar target information is based on image processing instead of directly working on the radar signal level. This may introduce additional error sources originating from mapping the radar target data from signal to image domain, but also yields the advantage of applying the proposed technique to most commercial radar systems by simply interfacing to the video output. To extract target candidates from the current radar image at time $k$, the following procedure is applied:

1. Masking the image to eliminate features of the user interface, e.g., colored heading lines, blob in center, radar information tables.

2. Conversion of the image from RGB to gray-scale (weighted average from color channels).

3. Blob detection with fixed range settings for convexity, circularity, inertia, size and intensity of expected targets.

4. Each detected target candidate per frame is expressed in range and bearing, relative to the position of the vessel carrying the radar.

The key aspect in this processing chain is certainly the scale-invariant blob detection to eventually detect target candidates. This algorithm is well described in the literature and finds many applications in image based target detection and tracking such as described in [20]. For this work the implementation provided by the OpenCV framework was used [21]. Figures 2a to 2c show the different radar processing stages.

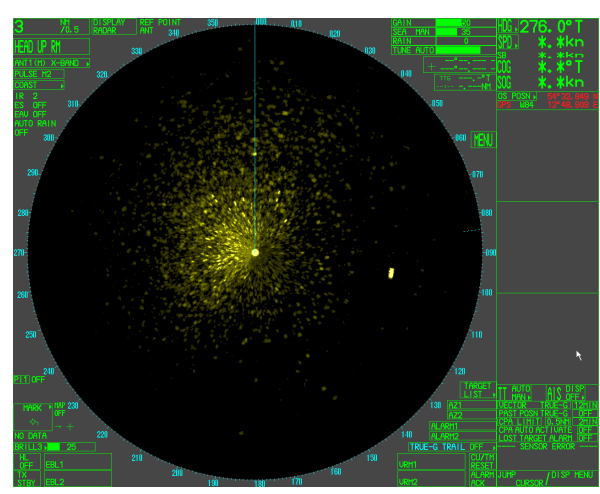

(a) Original radar image.

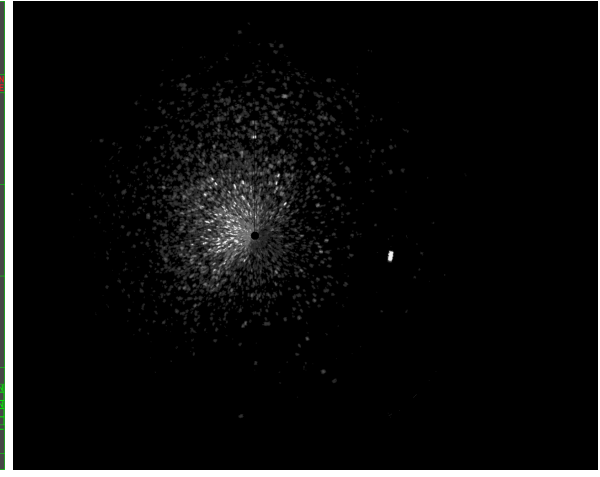

(b) Image after background subtraction and (c) Extracted target candidates (red circles) at gray-scale conversion.

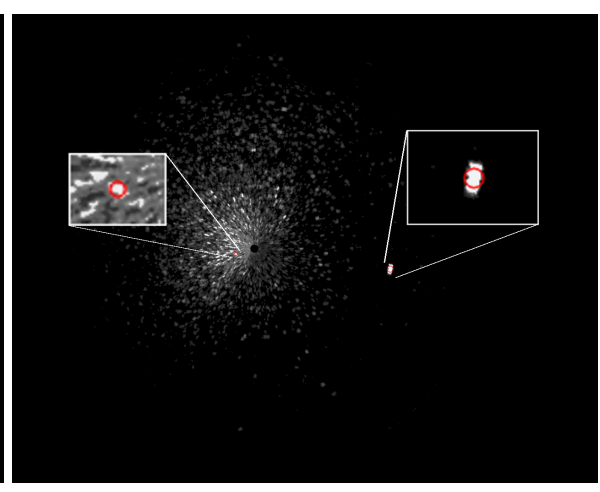

time $k$ after blob detection.

Figure 2: Processing chain for one radar image at time $k$ to extract the target candidates.

The set of extracted radar measurements is defined as $\mathcal{Z}_{k}^{s}=\left\{\mathbf{z}_{k}^{1}, \ldots, \mathbf{z}_{k}^{M}\right\}$, with the $j^{\text {th }}$ measurement vector $\mathbf{z}_{k}^{j}=\left[z_{k}^{r}, z_{k}^{b}\right]^{T}$ comprising range and bearing of the target candidate. The state update of $\mathbf{x}_{k \mid k-1}^{i}$ conditioned on the associated radar target measurements is based on the definition of $h^{s}\left(\mathbf{x}_{k \mid k-1}^{i}, \varepsilon_{k}^{r, s}\right)$ from Eq. 2 given as

$$
h^{s}\left(\mathbf{x}_{k \mid k-1}^{i}, \boldsymbol{\epsilon}_{k}^{r}\right)=\left[\begin{array}{c}
\sqrt{\left(p_{e, k \mid k-1}-p_{e}^{s}\right)^{2}+\left(p_{n, k \mid k-1}-p_{n}^{s}\right)^{2}} \\
\arctan \left(\frac{p_{n, k \mid k-1}-p_{n}^{s}}{p_{e, k \mid k-1}-p_{e}^{s}}\right)
\end{array}\right]+\boldsymbol{\epsilon}_{k}^{r},
$$


for $s=$ radar and with $\left\{p_{e}^{s}, p_{n}^{s}\right\}$ being the 2D reference coordinates of the radar system in the ENU frame of the tracked vessel. The sensor uncertainty in range $\left(\sigma_{r}\right)$ and bearing $\left(\sigma_{b}\right)$ is modeled in

$$
\mathbf{R}_{k}^{\text {radar }}=\left[\begin{array}{cc}
\sigma_{r}^{2} & 0 \\
0 & \sigma_{b}^{2}
\end{array}\right] .
$$

\section{AIS target data}

A typical AIS data set contains numerous static and dynamic parameters, that are distributed over different AIS message types and specified in the ITU-R recommendation [22]. The set of dynamic parameters always comprises the vessel position in longitude and latitude, COG and SOG, but may also contain true heading and rate of turn (ROT) information. To integrate the AIS data to the tracking process we simply assign the AIS message to the $t^{t h}$ target via its unique Maritime Mobile Service Identity (MMSI). Instead of applying a probabilistic association scheme we set up a validation gate around the predicted target position in the measurement domain to reject outliers from AIS data. For state correction the AIS coordinates are converted from WGS84 to the target's ENU frame that originates at the latest estimated target position in the global ECEF frame. This implies the AIS measurement vector $\mathbf{z}_{k}$ to be defined as $\left[z_{k}^{e}, z_{k}^{n}\right]^{T}$ and the corresponding measurement model from Eq. 2 becomes

$$
h^{s}\left(\mathbf{x}_{k \mid k-1}^{t}, \boldsymbol{\epsilon}_{k}^{r}\right)=\left[\begin{array}{c}
p_{e, k \mid k-1} \\
p_{n, k \mid k-1}
\end{array}\right]+\boldsymbol{\epsilon}_{k}^{r},
$$

with $s=A I S$ and the sensor noise in East and North coordinates being captured in

$$
\mathbf{R}_{k}^{A I S}=\left[\begin{array}{cc}
\sigma_{p, e}^{2} & 0 \\
0 & \sigma_{p, n}^{2}
\end{array}\right] .
$$

Note, that the update rate of AIS position reports is varying with the vessel's dynamics (see [22]). This also implies that the AIS measurements are in principle asynchronous to measurements obtained from radar; we were grabbing images at a rate of $0.5 \mathrm{~Hz}$.

\section{RESULTS FROM A MARITIME GNSS JAMMING EXPERIMENT}

In this section we will test the performance of the designed IMM-JPDA filter based on measurement data. As we are specifically interested in the resilience of the proposed scheme against AIS outages a challenging scenario was chosen involving two fast maneuvering vessels exposed to a strong GNSS interference.

\section{Measurement campaign setup}

In October 2015, a dedicated measurement campaign was conducted for two days in the Baltic Sea covering different scenarios of interest. The entire setup of the campaign is depicted in Figure 3. For evaluation purposes we selected a 1:15 h long snippet that exposed a test case of specific interest to us. This scenario can be characterized as follows:

- Three vessels were involved in total

$\circ$ two chartered ships, BALTIC TAUCHER II (offshore supply vessel) and AARON (tug vessel)

$\circ$ one external ship, NEUSTRELITZ (law enforcement vessel)

- BALTIC TAUCHER II performed highly dynamic maneuvering trials, being additionally equipped with an on-board multifrequency, multi-constellation GNSS receiver.

- AARON was anchored monitoring her proximity by her radar.

- AARON also acted as interferer to GPS carrying two common-off-the-shelf GNSS jammers, so-called Personal Privacy Devices (PPDs).

Note, that the NEUSTRELITZ was not under guidance by any of our team members, as she just entered the scene on her own behalf. This also implies that in contrast to the BALTIC TAUCHER II we were not able to generate a reference trajectory for the NEUSTRELITZ, due to the lack of high precision position data. For that reason, we can only test our algorithm on a qualitative scale, focusing on track completeness and the ability to separate targets. However, a quantitative measure for performance evaluation based on a metric, e.g., the Optimal Subpattern Assignment (OSPA) metric introduced in [23] cannot be given. 


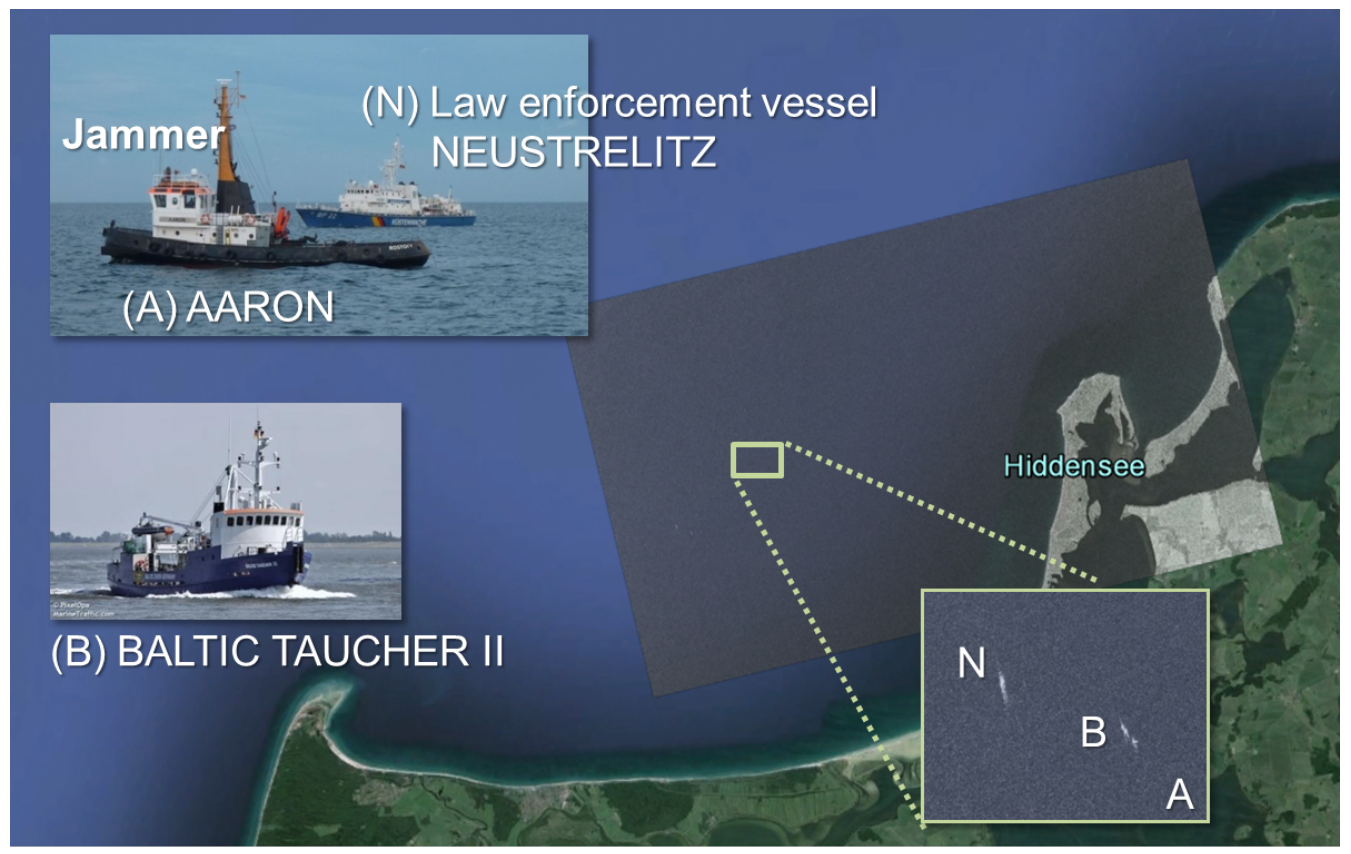

Figure 3: The measurement campaign was conducted in the Baltic Sea close to Hiddensee, Germany. Three vessels were involved, the BALTIC TAUCHER II (B), the AARON (A) and the law enforcement vessel NEUSTRELITZ (N). A SAR image is integrated showing all tree ships during the campaign.

\section{Impact of GPS jamming on the AIS}

Two commercial GNSS jammers were installed on-board the AARON, one low power device interfering only with GPS L1 and L2 band and one high power unit jamming GPS L1, L2, but also GSM and WiFi frequency bands (only GPS bands were used). The second device was observed to radiate with an output power approximately $10 \mathrm{~dB}$ higher than the first jammer. Both devices are depicted in Figures $4 \mathrm{a}$ and $4 \mathrm{~b}$. It is worth mentioning that the deployed jammers only affected the content of AIS messages, i.e., the positions obtained from Global Positioning System (GPS). The AIS transmission within the VHF bands remained untouched.

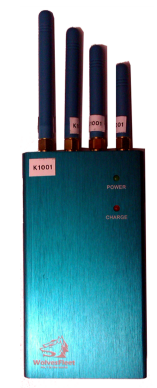

(a) Jammer LP.

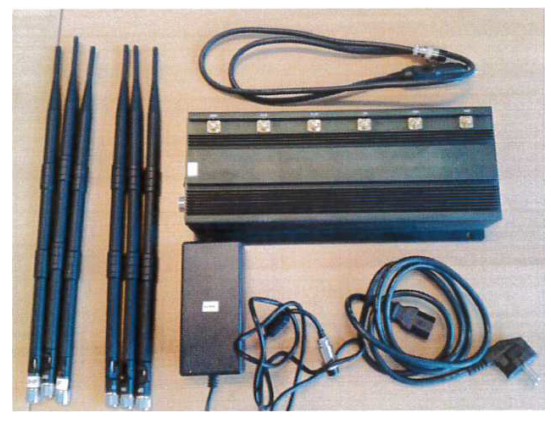

(b) Jammer HP.

Figure 4: Low and high power GPS jammers used in open sea field trials.

In case on-board GPS receivers fail to compute position data due the loss of lock it was questionable how this is treated within the broadcasted AIS messages. It turns out that the AIS transmitters of the received vessels are switching immediately to default mode in case the attached GPS receiver fails to provide a new position solution. This means, for the entire time the receiver was affected by GNSS interference the longitude and latitude information is set to $181^{\circ}$ and $91^{\circ}$. Although these values can easily be excluded from the fusion process, it also implies that the AIS is rendered useless for the tracking task.

In Figures $5 \mathrm{a}$ and $5 \mathrm{~b}$ the distance between each of the maneuvering vessels to the GNSS jammer is plotted, based on their transmitted AIS messages. On a second scale the ON/OFF times for both jammers in use are also marked. From these plots two phenomena become visible:

1. Only the law enforcement vessel NEUSTRELITZ was affected by any of the GNSS jammers, whereas BALTIC TAUCHER II hardly shows any influence. 


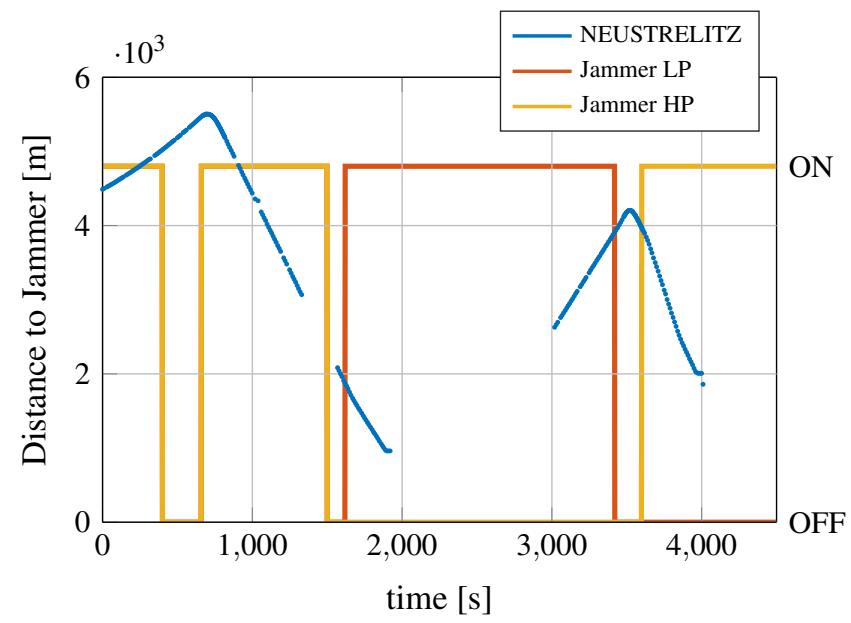

(a) Distance NEUSTRELITZ to AARON against jammers being switched ON/OFF.

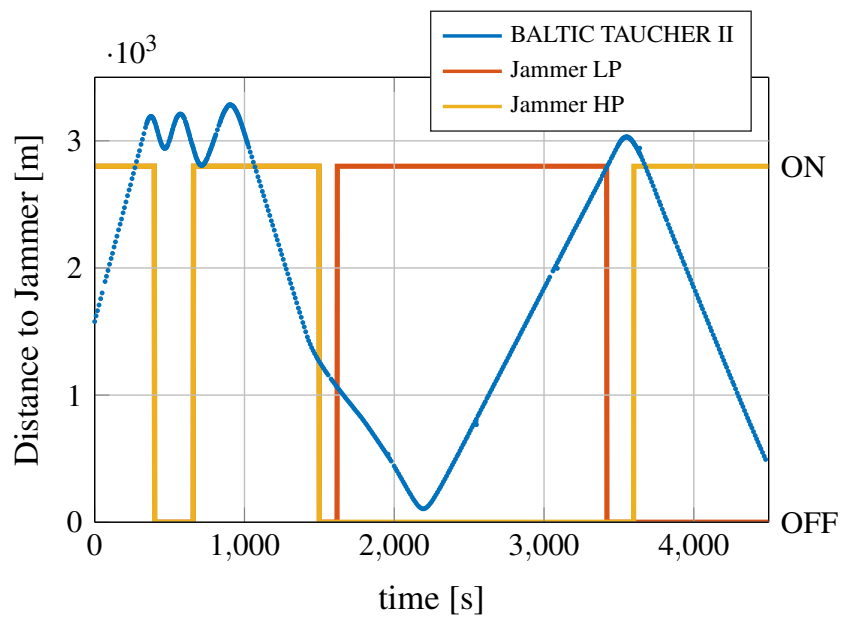

(b) Distance BALTIC TAUCHER II to AARON against jammers being switched ON/OFF.

Figure 5: The plots show the distance between each of the vessels to the AARON, i.e., the GNSS jammer, over time (dotted blue lines). On top of that the ON/OFF times of the low- and high-power devices are also depicted (red and yellow curves).

2. Considering the law enforcement vessel, the GPS outages clearly depend on the distance to the jammer, starting roughly from $3000 \mathrm{~m}$ downwards. However, additional effects like the tilt of the jamming vessel may have caused an interruption of the Line of Sight (LoS) between maneuvering vessel and jammer (see re-established position solutions between $1560 \mathrm{~s}$ and $1920 \mathrm{~s}$ in Figure 5a).

While the second of the above statements is not very surprising, the fact that the equipment of BALTIC TAUCHER II was not affected by the jammers did not meet the expectations. We can only speculate about the reasons for this: Most likely the corresponding GPS receiver was not affected due to the specific signal characteristics of the utilized jammers; saw-tooth-like chirp signals were transmitted. Another possibility would be that the receiver's antenna was shielded from the interfering signal by the monkey deck of the ship. Regardless of these considerations, a very challenging scenario was captured for further evaluation of the proposed tracking scheme imposing not only continuous AIS outages but also false radar measurements to the filter.

\section{Tracking performance}

The trajectories of both vessels obtained from AIS measurements are depicted in Figure 6 with red and blue cross markers. The reference position of AARON being equipped not only with the jammers but also with the observing radar is pictured as ocher pentagon. In principle, wee see the same plot as in Figure 5, only that the scenario is now transferred to the spatial domain plotting all accumulated AIS position coordinates over time. The challenge for the IMM-JPDA filter is clearly to overcome the AIS outages of the red curve by incorporating radar measurements only. All of these measurements accumulated frame by frame from radar images are depicted in Figure 7 . We can clearly identify the two maneuvering target tracks, a cloud of clutter measurements south-west of the AARON, and at least three nearly static objects that were detected repeatedly at ENU coordinates $\sim\{(-2060 \mathrm{~m}, 0 \mathrm{~m}),(0 \mathrm{~m}, 1430 \mathrm{~m}),(960 \mathrm{~m}, 0 \mathrm{~m})\}$. In fact, those three objects were buoys in the surrounding of the campaign area. The targets within the IMM-JPDA filter were initialized with the first obtained AIS position report being transmitted from any of the two maneuvering vessels within the considered time frame. Afterwards, the computation of the joint probabilities were triggered on every new set of radar target candidate measurements, whereas new arriving AIS position reports were directly associated to the corresponding target as was described above. However, a new iteration of the IMM scheme as depicted in Figure 1 is triggered on any sensor measurement used for target state update. The parameterization of the filter with respect to measurement and sensor noise covariances is summarized in Table 1.

The filtered target tracks over the full time span can be observed in Figure 8 plotted again in the spatial domain. Clearly, two complete tracks were resolved, i.e., neither track coalescence nor flipped track assignments were observed. The two latter statements are especially important for the time of GNSS jamming, in which only the radar measurements are used to update the state of target 2 (law enforcement vessel NEUSTRELITZ). During these times of AIS outages the NEUSTRELITZ was passing the area exposed to a high number of clutter measurements in radar. Remarkably, even under these severe conditions the target was tracked completely. 


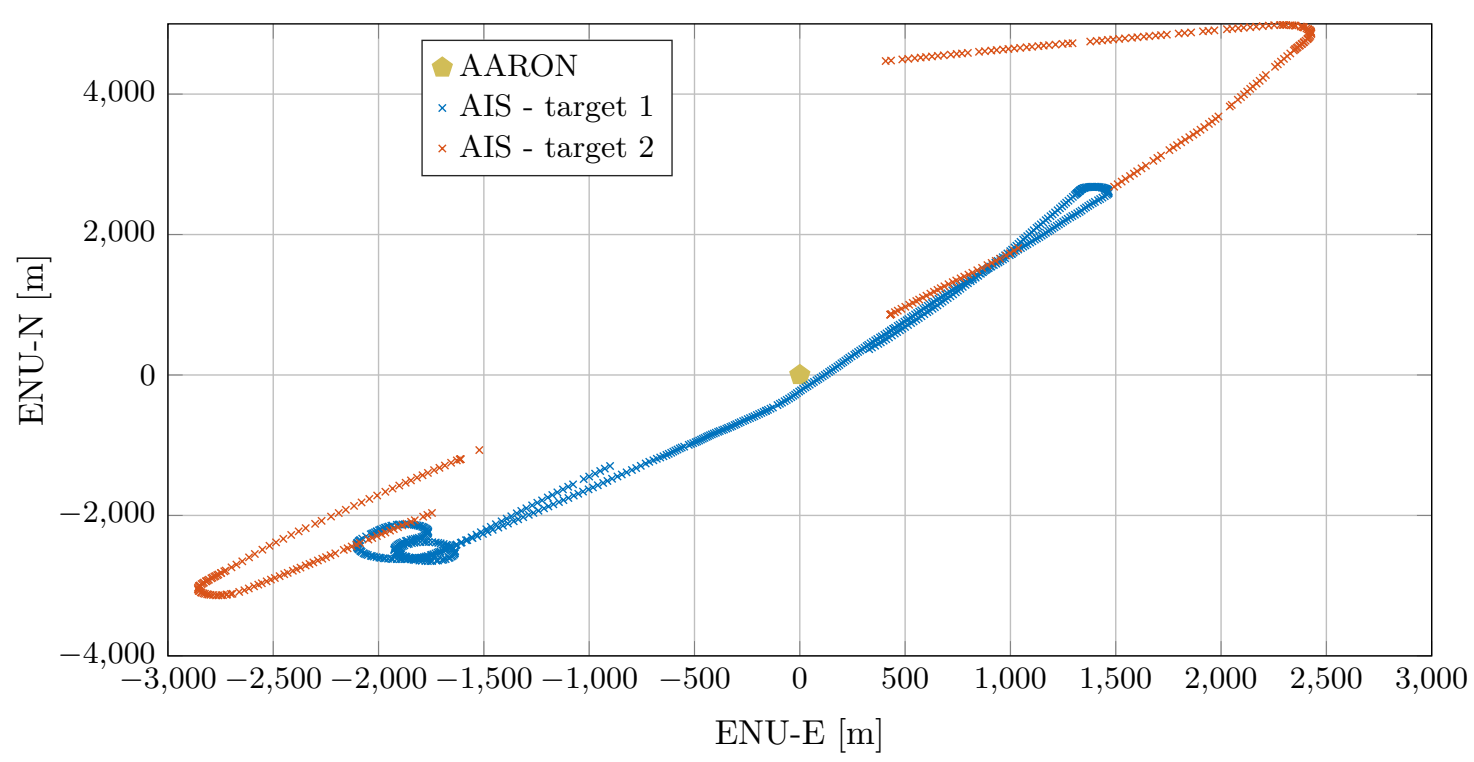

Figure 6: AIS position reports of both maneuvering targets plotted in the ENU frame originating at the global position of the AARON (ocher pentagon). The AIS target 2 (red marks) clearly exhibits outages during times of GNSS jamming.

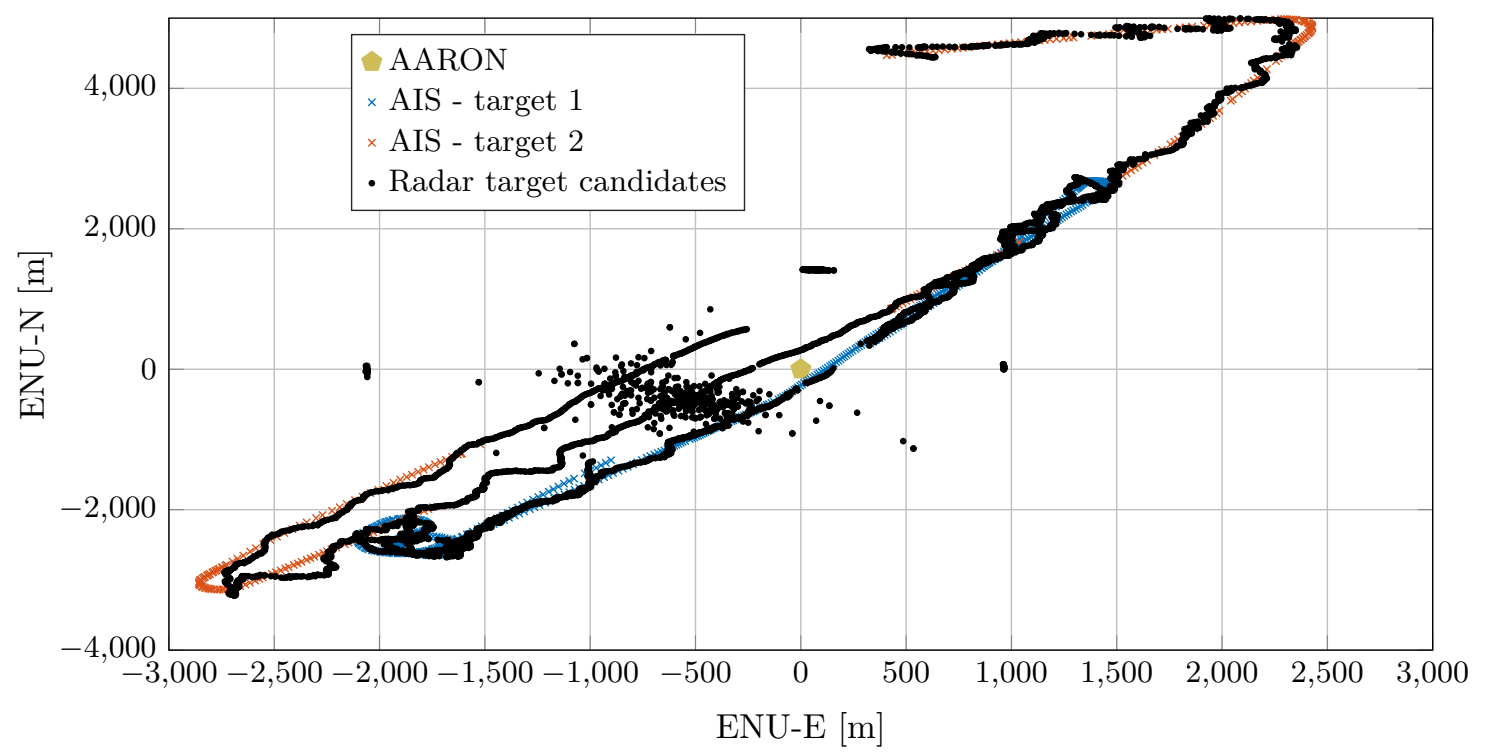

Figure 7: Extracted radar target candidates plotted on top of the AIS position reports. The pitching and rolling of the AARON, being equipped with the radar system, is not compensated and therefore projected into the target trajectories.

Table 1: Summary of IMM-JPDA filter settings.
(a) Dynamic model dependent settings.
(b) Sensor model dependent settings.
(c) General JPDA settings.

(d) IMM transition

\begin{tabular}{|c|c|c|c|c|c|}
\hline & model uncertainty $(\mathbf{Q})$ & & sensor uncertainty $(\mathbf{R})$ & JPDA type & parametric \\
\hline \multirow{2}{*}{$\mathrm{CV}$} & $\sigma_{v}=0.01 \mathrm{~m} / \mathrm{s}^{2}$ & AIS & $\sigma_{p, e}=5 \mathrm{~m}$ & spatial density $\lambda$ & $10^{-9}$ \\
\hline & $\sigma_{\psi}=0.01^{\circ} / \mathrm{s}$ & דונד & $\sigma_{p, n}=5 \mathrm{~m}$ & \multirow{2}{*}{$P_{d, r a d a r}$} & \multirow[t]{2}{*}{0.8} \\
\hline CTRV & $\begin{array}{l}\sigma_{v}=0.1 \mathrm{~m} / \mathrm{s}^{2} \\
\sigma_{\omega}=0.1^{\circ} / \mathrm{s}^{2}\end{array}$ & radar & $\begin{array}{l}\sigma_{r}=50 \mathrm{~m}, \\
\sigma_{b}=0.1 \mathrm{rad}\end{array}$ & & \\
\hline
\end{tabular}
probabilities [12].

$\mathbf{T}=\left[\begin{array}{ll}0.9 & 0.1 \\ 0.1 & 0.9\end{array}\right]$ 


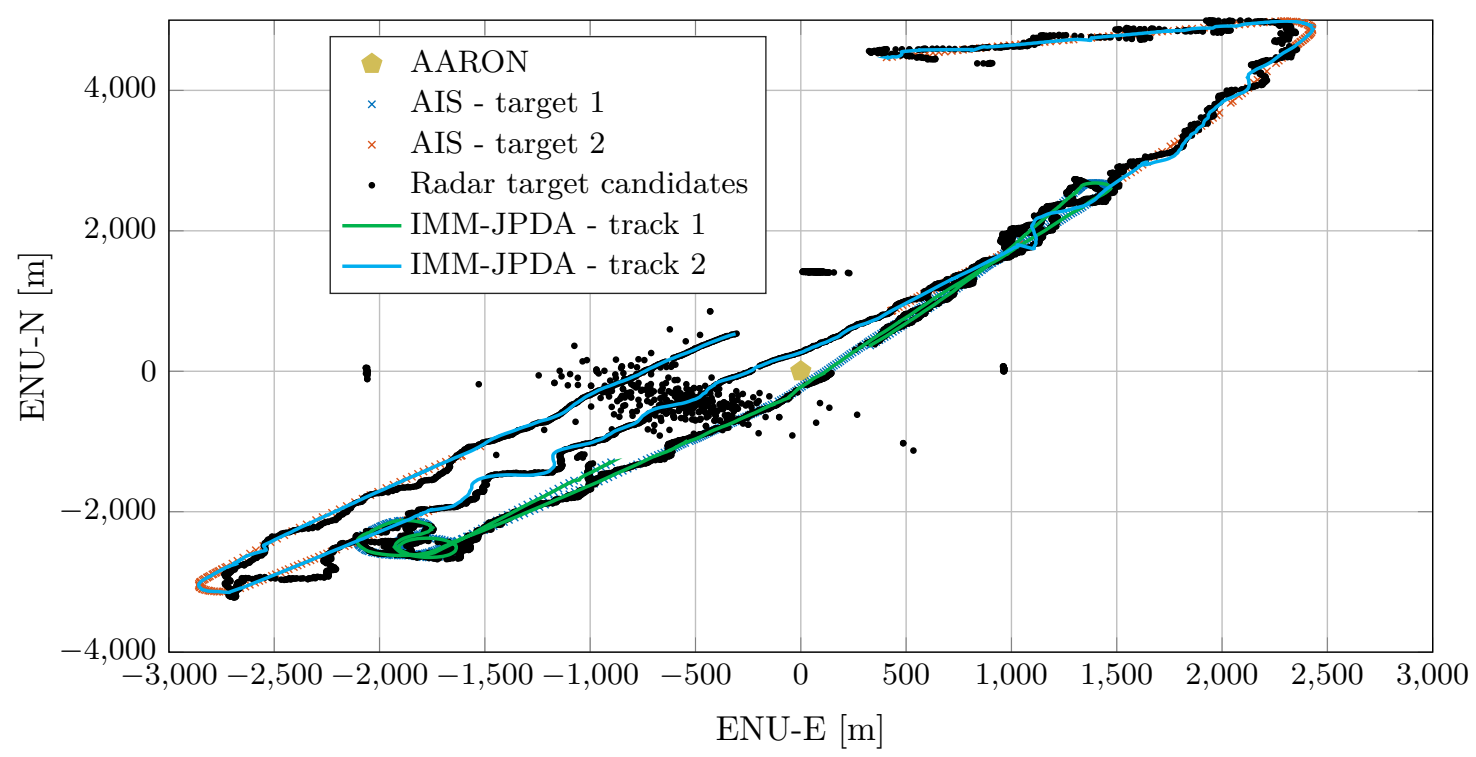

Figure 8: Resulting tracks from fusing AIS and radar in the designed IMM-JPDA filter for a multi-target scenario. The AIS outages due to the jammed GPS signals are clearly compensated in solid blue and green lines (IMM-JPDA track 1 and 2).

\section{Discussion}

Due to the lack of reference data no quantitative analysis of the proposed filter was possible within this study. Nevertheless, the results obtained from the maritime GNSS jamming experiment are already very promising with respect to our main objectives. Despite continuous AIS outages the filter was able to generate complete tracks for both targets even throughout clutter measurements, i.e., it appears to be more robust to AIS outages. It can be concluded, that the filtered tracks yield a more complete and reliable picture of the current traffic situation compared to the individual sensors, which was in fact the main intention of this work. Naturally, there are also some limitations to the proposed scheme, which shall be discussed in the following. First of all, the overall accuracy of the filtered objects is bounded by the AIS, which is in that respect a magnitude better sensor (see Table 1b). Again, this is expected and tolerable, since track completeness is of greater interest than track accuracy for maritime situation awareness. Secondly, the uncertainty in range and bearing within the radar measurement model was set to rather high values compared to other common-off-the-shelf marine radars. This was done intentionally to compensate for the motion of the AARON, which had the radar system mounted on her mast top. Despite being anchored, the AARON was still subject to the motion of the waves, i.e., the assumption of a static system was actually violated. The resulting pitching and rolling of the AARON, however, is projected onto the trajectories of the maneuvering vessels, as can be seen in the harmonic structure within the extracted target candidates in Figure 7. In case this motion was measured and compensated, e.g., with an additional inertial sensor on-board, the radar uncertainty could be tightened to more realistic values. Thirdly, the current framework is not capable of initializing targets based on radar measurements. This feature would require either the extension of the algorithm by deploying a classical $M / N$ detection rule or to move to a more generic MTT framework. And last but not least, the applicability of the standard JPDA filter clearly depends on the scenario; on the number of (clutter) measurements and targets to be tracked to be precise. In the demonstrated case of only two targets and a rather conservative setting for the radar target extraction procedure the computation of the joint probabilities is not problematic. However, this may change drastically for different scenarios due to the combinatoric explosion of association events. Thus, a more generic framework also capable of large scale traffic monitoring may require different MTT approaches, e.g., following variations of the JPDA or MHT instead.

\section{CONCLUSION}

In this work, we presented an approach to improve the reliability of on-board maritime traffic situation assessment in the presence of GNSS jamming rendering the maritime Automatic Identification System useless. An IMM-JPDA filter was designed for multi-target tracking fusing the Automatic Identification System with a single radar. The evaluation of this filter was based on measurement data obtained from a dedicated maritime GNSS jamming campaign in the Baltic Sea. This field trial included two maneuvering vessels and one static ship, where the latter carried two GPS jammers and the radar system for traffic monitoring. Despite AIS outages and radar clutter measurements the filter showed good performance in terms of track completeness and track separation. The accuracy of the filtered tracks, however, could not have been determined as a consequence to lacking reference data. In future work we will direct our research towards cooperative schemes for maritime traffic situation assessment aiming at a network of spatially distributed sensor nodes or vessels, respectively. By exploiting the diverse information within such a 
network, e.g., the variety of radar aspect angles, we expect a significant improvement; not only in robustness against threats like GNSS jamming or intentional misuse of the AIS, but also in the accuracy of the assessed situation picture. In fact, a reliable and complete situation picture is key to many high level interpretations, such as collision avoidance or anomaly detection.

\section{ACKNOWLEDGEMENT}

We wish to thank our colleagues at DLR Ralf Ziebold, Stefan Gewies and Uwe Netzband for thoroughly preparing and carrying out the measurement campaign that provided the basic data set to this work. We also gratefully appreciate the insightful discussions with our former colleague Michailas Romanovas from Baselabs GmbH.

\section{REFERENCES}

[1] United Nations, World Economic Situation and Prospects 2012, ch. 2, pp. 41-66. United Nations publication, 2012.

[2] M. Balduzzi, A. Pasta, and K. Wilhoit, "A Security Evaluation of AIS Automated Identification System," in Proceedings of the 30th annual computer security applications conference, ASAC, (New Orleans, LA, USA), December 2014.

[3] L. P. Perera, V. Ferrari, F. P. Santos, M. A. Hinostroza, and C. G. Soares, "Experimental Evaluations on Ship Autonomous Navigation and Collision Avoidance by Intelligent Guidance," IEEE JOURNAL OF OCEANIC ENGINEERING, vol. 40, APRIL 2015.

[4] P. Braca, M. Vespe, S. Maresca, and J. Horstmann, "A Novel Approach to High Frequency Radar Ship Tracking Exploiting Aspect Diversity," Geoscience and Remote Sensing Symposium (IGARSS), 2012 IEEE International, pp. 6895 - 6898, 2012.

[5] M. Guerriero, P. Willett, S. Coraluppi, and C. Carthel, "Radar/AIS Data Fusion and SAR tasking for Maritime Surveillance," in International Conference on Information Fusion, vol. 11th, 2008.

[6] F. Mazzarella and M. Vespe, "SAR Ship Detection and Self-Reporting Data Fusion Based on Traffic Knowledge," IEEE GEOSCIENCE AND REMOTE SENSING LETTERS, April 2015.

[7] Y. Bar-Shalom, F. Daum, and J. Huang, "The Probabilistic Data Association Filter," IEEE CONTROL SYSTEMS MAGAZINE, December 2009.

[8] C. Kim, F. Li, A. Ciptadi, and J. M. Rehg, "Multiple hypothesis tracking revisited," in 2015 IEEE International Conference on Computer Vision (ICCV), pp. 4696-4704, Dec 2015.

[9] R. Mahler, “,statistics 102,,for multisource-multitarget detection and tracking,” IEEE Journal of Selected Topics in Signal Processing, vol. 7, pp. 376-389, June 2013.

[10] R. Mahler, "A brief survey of advances in random-set fusion," in Control, Automation and Information Sciences (ICCAIS), 2015 International Conference on, pp. 62-67, Oct 2015.

[11] G. W. Pulford, “Taxonomy of multiple target tracking methods," IEEE Proceedings - Radar, Sonar and Navigation, vol. 152, pp. 291-304, October 2005.

[12] H. A. P. Blom and Y. Bar-Shalom, "The Interacting Multiple Model Algorithm for Systems with Markovian Switching Coefficients," IEEE Transactions on Automatic Control, vol. 33, 1988.

[13] H. A. P. Blom and E. A. Bloem, "Combining imm and jpda for tracking multiple maneuvering targets in clutter," in Information Fusion, 2002. Proceedings of the Fifth International Conference on, vol. 1, pp. 705-712 vol.1, July 2002.

[14] J. K. Tugnait, "Tracking of multiple maneuvering targets in clutter using multiple sensors, imm and jpda coupled filtering," in American Control Conference, 2003. Proceedings of the 2003, vol. 2, pp. 1248-1253, June 2003.

[15] T. Fortmann, Y. Bar-Shalom, and M. Scheffe, "Sonar tracking of multiple targets using joint probabilistic data association," IEEE Journal of Oceanic Engineering, vol. 8, pp. 173-184, Jul 1983.

[16] M. Schuster, M. Blaich, and J. Reuter, "Collision Avoidance for Vessels using a Low-Cost Radar Sensor," The International Federation of Automatic Control, pp. 9673 - 9678, 2014.

[17] G. Siegert, P. Banyś, C. S. Martínez, and F. Heymann, "EKF Based Trajectory Tracking and Integrity Monitoring of AIS Data," in IEEE/ION Position, Location and Navigation Symposium - PLANS, (Savannah, GA), pp. 887 - 897, IEEE, April 2016.

[18] J. D. Glass, W. D. Blair, and Y. Bar-Shalom, "IMM Estimators with Unbiased Mixing for Tracking Targets Performing Coordinated Turns," Proceedings IEEE Aerospace Conference, 2013.

[19] S. J. Julier and J. K. Uhlmann, "A New Extension of the Kalman Filter to Nonlinear Systems," in Proc. of AeroSense: The 11th Int. Symp. on Aerospace/Defence Sensing, Simulation and Controls., pp. 182-193, 1997.

[20] M. Isard and J. MacCormick, "BraMBLe: A Bayesian multiple-blob tracker," in Eighth IEEE International Conference on Computer Vision, vol. 2, pp. 34-41, 2001.

[21] "OpenCV 3.1.0." https://github.com/Itseez/opencv.git. Accessed: 2016-12-01.

[22] ITU Radiocommunication Sector (ITU-R), "Technical characteristics for an automatic identification system using time division multiple access in the VHF maritime mobile band.," Recommendation M.1371-5, ITU, February 2014.

[23] D. Schuhmacher, B. T. Vo, and B. N. Vo, "On performance evaluation of multi-object filters," in Information Fusion, 2008 11th International Conference on, pp. 1-8, June 2008. 